\title{
Subsonic interface crack with crack face contact
}

\author{
Shaohua Chen ${ }^{a}$, Guang $\mathrm{Xu}^{\mathrm{b}}$, Cong Yan ${ }^{\mathrm{c}}$ \\ LNM, Institute of Mechanics, Chinese Academy of Sciences, Beijing, 100080, China \\ a Chenshaohua72@hotmail.com, ${ }^{b}$ xu_g10@163.com, ${ }^{c}$ ycqqren@gmail.com
}

Keywords: Subsonic crack, bimaterial interface, linear contact model, singularity

\begin{abstract}
A steady-state subsonic interface crack propagating between an elastic solid and a rigid substrate with crack face contact is studied. Two cases with respective to the contact length are considered, i.e., semi-infinite and finite crack face contact. Different from a stationary or an open subsonic interface crack, stress singularity at the crack tip in the present paper is found to be non-oscillatory. Furthermore, in the semi-infinite contact case, the singularity of the stress field near the crack tip is less than $1 / 2$. In the finite contact case, no singularity exists near the crack tip, but less than $1 / 2$ singularity does at the end of the contact zone. In both cases, the singularity depends on the linear contact coefficient and the crack speed. Asymptotic solutions near the crack tip are given and analyzed. In order to satisfy the contact conditions, reasonable region of the linear contact coefficient is found. In addition, the solution predicts a non-zero-energy dissipation rate due to crack face contact.
\end{abstract}

\section{Introduction}

Cracks in homogeneous solids or along bimaterial interfaces play an important role in determining the behavior of materials and structures. Generally, the fracture toughness and the energy release rate are obtained under the non-contact conditions of crack faces, which is violated in many real-life situations. For examples: cracks lie along a bimaterial interface with dominated shear loadings, sizeable contact zones emerge near the crack tip. Crack face contact does exist in earthquake. On the other hand, the asymptotic elastic fields of not only a stationary but also a dynamic interface crack possess oscillatory singularity [1-3] (Williams, 1959; Wu, 1991; Yang et al. 1991), and their behaviors near the tip are physically inadmissible because crack faces will interpenetrate each other due to the oscillatory.

A series of experiments [4-5] have been done several years before. These experiments also show that a relatively large contact zone exists behind the crack tip. The contact zone length is between 1.5-2 $\mathrm{mm}$ in the shear dominated intersonic interface fracture [5]. Crack face contact has also been observed in numerical simulations of Xu and Needleman [6]. In order to understand the stress and deformation fields around propagating interface cracks with crack face contact, many works have been done. Deng [7] investigated the asymptotic features of semi-infinite contact for sub-Rayleigh interface crack growing steadily along the interface of two dissimilar, homogeneous, isotropic and linearly elastic solids. As for the intersonic crack face contact problem, Huang, et al.'s solution [8] features a large scale contact zone and two distinct traveling shock waves, one emanating from the crack tip and the other from the end of the contact zone.

Motivated by the aforementioned experimental and theoretical observations, this paper presents an investigation of the stress and displacement fields for a subsonic propagating interface crack with semi-infinite and finite crack face contacts. Since an elastic/rigid bimaterial system [8] can capture all characteristic features of a general elastic/elastic bimaterial [9] and the solution of the former is significantly simpler than that of the latter, an elastic/rigid bimaterial system will be considered in the present paper, which will provide basic solutions for further use. 


\section{General formula for subsonic propagating interface crack}

A general formulation for a subsonic crack propagating along an interface between an elastic solid and a rigid substrate under in-plane (plane strain or plane stress) deformation is given in this section. The model is shown in Fig. 1, in which the interface lies in $\eta_{1}$ axis. The crack tip lies at the origin of the coordinate system $\left(\eta_{1}, \eta_{2}\right)$ and the crack tip propagates in the positive $\eta_{1}$ direction with a constant speed $v, v<C_{s}$, where $C_{s}$ is the shear wave speed of the elastic solid. The crack growth is assumed to be steady-state. The equations of motion for the elastic solid can be expressed by two displacement potentials $\phi, \psi$,

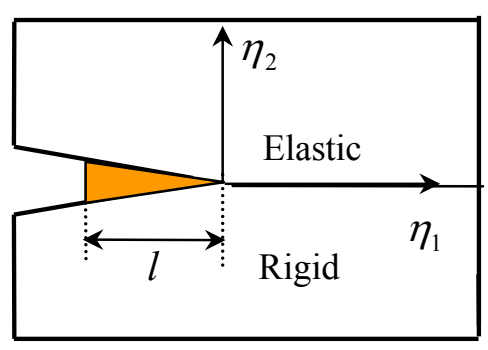

Fig.1 A subsonic interface crack propagating between an elastic solid and a rigid substrate.

$$
\left\{\begin{array}{l}
\phi_{, 11}+\frac{1}{\alpha_{l}^{2}} \phi_{, 22}=0 \\
\psi_{, 11}+\frac{1}{\alpha_{s}^{2}} \psi_{, 22}=0
\end{array}, \eta_{2}>0, \alpha_{l}=\sqrt{1-\frac{v^{2}}{C_{l}^{2}}}, \alpha_{s}=\sqrt{1-\frac{v^{2}}{C_{s}^{2}}}\right.
$$

where $C_{l}$ is the longitudinal wave speed of the elastic solid.

The general solutions can be expressed by two analytical functions as follows,

$$
\phi=\operatorname{Re}\left[F\left(z_{l}\right)\right] \quad \psi=\operatorname{Im}\left[G\left(z_{s}\right)\right] \quad \eta_{2}>0
$$

where $F\left(z_{l}\right)$ and $G\left(z_{s}\right)$ are analytical functions of $z_{l}$ and $z_{s}$ in

the upper half plane, respectively. $z_{l}=\eta_{1}+i \alpha_{l} \eta_{2}$ and $z_{s}=\eta_{1}+i \alpha_{s} \eta_{2}$.

The in-plane displacements $u_{1}, u_{2}$, and the stress components in the elastic solid can be expressed by the two analytical functions as

$$
\left\{\begin{array}{l}
u_{1}=\operatorname{Re}\left[F^{\prime}\left(z_{l}\right)+\alpha_{s} G^{\prime}\left(z_{s}\right)\right] \\
u_{2}=-\operatorname{Im}\left[\alpha_{l} F^{\prime}\left(z_{l}\right)+G^{\prime}\left(z_{s}\right)\right]
\end{array}\right.
$$

$$
\left\{\begin{array}{l}
\sigma_{11}=\mu \operatorname{Re}\left[\left(1+2 \alpha_{l}^{2}-\alpha_{s}^{2}\right) F^{\prime \prime}+2 \alpha_{s} G^{\prime \prime}\right] \\
\sigma_{22}=-\mu \operatorname{Re}\left[\left(1+\alpha_{s}^{2}\right) F^{\prime \prime}+2 \alpha_{s} G^{\prime \prime}\right] \\
\sigma_{12}=-\mu \operatorname{Im}\left[2 \alpha_{l} F^{\prime \prime}+\left(1+\alpha_{s}^{2}\right) G^{\prime \prime}\right]
\end{array}\right.
$$

where $\mu$ is the shear modulus of the elastic solid.

It is convenient to define the stress components as a traction vector,

$$
\mathbf{t}=\left\{\begin{array}{l}
\sigma_{12} \\
\sigma_{22}
\end{array}\right\}=2 \operatorname{Re}\left[\mathbf{h}^{\prime}(z)\right] \quad \mathbf{h}(z)=\frac{\mu}{2}\left[\begin{array}{cc}
2 i \alpha_{l} & i\left(1+\alpha_{s}^{2}\right) \\
-\left(1+\alpha_{s}^{2}\right) & -2 \alpha_{s}
\end{array}\right]\left\{\begin{array}{l}
F^{\prime}\left(z_{l}\right) \\
G^{\prime}\left(z_{s}\right)
\end{array}\right\}
$$

and the displacement vector is defined as

$$
\mathbf{u}=\left\{\begin{array}{l}
u_{1} \\
u_{2}
\end{array}\right\}=2 \operatorname{Im}[\mathbf{B h}(z)] \quad \mathbf{B}=\left[\begin{array}{cc}
Q & -i P \\
i P & R
\end{array}\right]
$$

where $z$ is replaced by $z_{l}$ in $F$ function and $z_{s}$ in $G$ function. The terms in matrix $\mathbf{B}$ are

$$
Q=\frac{\alpha_{s}\left(1-\alpha_{s}^{2}\right)}{\mu D} \quad P=\frac{2 \alpha_{l} \alpha_{s}-\left(1+\alpha_{s}^{2}\right)}{\mu D} \quad R=\frac{\alpha_{l}\left(1-\alpha_{s}^{2}\right)}{\mu D} \quad D=4 \alpha_{s} \alpha_{l}-\left(1+\alpha_{s}^{2}\right)^{2}
$$




\section{Semi-infinite contact case}

The semi-infinite contact problem is considered first. At the interface, $\eta_{1}>0, \eta_{2}=0$, the displacements should vanish due to a rigid substrate,

$\mathbf{u}=0$

From equation (5), the above equation can be written as

$\mathbf{B h}^{+}-\overline{\mathbf{B}} \overline{\mathbf{h}}^{-}=0$

Based on analytical continuation, a new analytical function $\boldsymbol{\theta}(z)$ is introduced as follows,

$$
\boldsymbol{\theta}(z)= \begin{cases}\mathbf{B h}(z) & \operatorname{Im}(z) \geq 0 \\ \overline{\mathbf{B}} \overline{\mathbf{h}}(z) & \operatorname{Im}(z)<0\end{cases}
$$

The function $\boldsymbol{\theta}(z)$ is analytical in the entire plane, except on the crack face $\left(\eta_{1}<0, \eta_{2}=0\right) . \boldsymbol{\theta}(z)$ is to be determined by the boundary conditions on the crack face. In the present study, linear contact model is used. The stress components and displacements within the contact zone are expressed as

$$
\sigma_{12}=\lambda \sigma_{22} \quad u_{2}=0 \quad \sigma_{22} \leq 0
$$

where $\lambda$ is the linear contact coefficient and assumed to be a constant.

In addition, the shear traction at the contact face should be resisting the relative sliding on the crack face, which means,

$$
-\sigma_{12} v_{1}<0
$$

where $v_{1}=\mathrm{d} u_{1} / \mathrm{d} t$ is the particle velocity along the crack face and $v_{1}=\mathrm{d} u_{1} / \mathrm{d} t=-v \partial u_{1} / \partial \eta_{1}$.

The first two equations in (10) can then be written as a Hilbert equation,

$$
\mathbf{U} \boldsymbol{\theta}^{\prime+}+\mathbf{V} \boldsymbol{\theta}^{\prime-}=0 \quad \eta_{1}<0
$$

where

$$
\mathbf{U}=\left[\begin{array}{cc}
R+\lambda P i & P i-\lambda Q \\
0 & 1
\end{array}\right] \quad \mathbf{V}=\left[\begin{array}{cc}
R-\lambda P i & -P i-\lambda Q \\
0 & -1
\end{array}\right]
$$

Solving the above Hilbert equation yields

$$
\boldsymbol{\theta}^{\prime}=\left\{\begin{array}{c}
\frac{\lambda Q}{R} A(z)+\frac{E(z)}{z^{r}} \\
A(z)
\end{array}\right\} \quad r=\frac{1}{2}+\frac{1}{\pi} \arctan \frac{\lambda P}{R}
$$

where $A(z)$ and $E(z)$ are two entire functions and analytical in the entire plane including the crack face. From equation (14), one can find the singularity of the stress field is $r(r \leq 1 / 2)$, which is influenced by the linear contact coefficient and the crack velocity.

The two analytical functions $F^{\prime \prime}(z)$ and $G^{\prime \prime}(z)$ can be obtained easily as well as the whole stress fields, from which the asymptotic stress fields near the crack tip are,

$$
\sigma_{11}=\frac{2 e_{0}}{D\left(Q R-P^{2}\right)}\left\{\frac{-\left(1+2 \alpha_{l}^{2}-\alpha_{s}^{2}\right)\left[2 \alpha_{s} R+\left(1+\alpha_{s}^{2}\right) P\right] \sin \left(\theta_{l} r\right)}{\xi_{l}^{r}}+\frac{2 \alpha_{s}\left[\left(1+\alpha_{s}^{2}\right) R+2 \alpha_{l} P\right] \sin \left(\theta_{s} r\right)}{\xi_{s}^{r}}\right\}
$$




$$
\begin{aligned}
& \sigma_{22}=\frac{2 e_{0}}{D\left(Q R-P^{2}\right)}\left\{\frac{\left(1+\alpha_{s}^{2}\right)\left[2 \alpha_{s} R+\left(1+\alpha_{s}^{2}\right) P\right] \sin \left(\theta_{l} r\right)}{\xi_{l}^{r}}-\frac{2 \alpha_{s}\left[\left(1+\alpha_{s}^{2}\right) R+2 \alpha_{l} P\right] \sin \left(\theta_{s} r\right)}{\xi_{s}^{r}}\right\} \\
& \sigma_{12}=\frac{2 e_{0}}{D\left(Q R-P^{2}\right)}\left\{\frac{2 \alpha_{s}\left[2 \alpha_{l} R+\left(1+\alpha_{s}^{2}\right) P\right] \cos \left(\theta_{l} r\right)}{\xi_{l}^{r}}-\frac{\left(1+\alpha_{s}^{2}\right)\left[\left(1+\alpha_{s}^{2}\right) R+2 \alpha_{l} P\right] \cos \left(\theta_{s} r\right)}{\xi_{s}^{r}}\right\}
\end{aligned}
$$

where we have used the following definitions,

$$
z_{l}=\eta_{1}+i \alpha_{l} \eta_{2}=\xi_{l} e^{i \theta_{l}} \quad z_{s}=\eta_{1}+i \alpha_{s} \eta_{2}=\xi_{s} e^{i \theta_{s}}
$$

and the entire functions $A(z)$ and $E(z)$ are expanded into Taylor series $A(z)=\sum_{n=0}^{\infty} a_{n} z^{n}$ and $E(z)=\sum_{n=0}^{\infty} e_{n} z^{n}$, all the coefficients $a_{n}, e_{n}$ are real. $e_{0}$ is the leading term in the Taylor expansion of $E(z)$, which is similar to the stress intensity factor in fracture mechanics. The real parameter $e_{0}$ represents the amplitude of the near-tip asymptotic field and depends on geometry of the bimaterial, time-varying external loading, and the crack tip velocity.

Due to a steady-state crack, the particle velocity on the

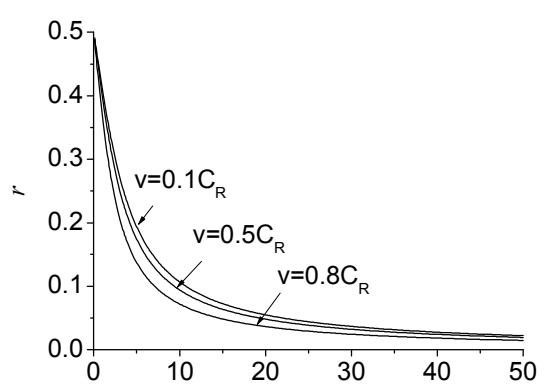

Fig. 2 The crack tip singularity via the linear contact coefficient contact crack face is

$$
v_{1}=-v \frac{\partial u_{1}}{\partial \eta_{1}}=\frac{2 v e_{0}}{\eta_{1}^{r}} \sin (\pi r)
$$

The requirement of the shear traction on the contact crack face resisting crack face sliding,

$$
-\sigma_{12} v_{1}=\frac{4 e_{0}^{2} R^{2} v \lambda P}{\left(Q R-P^{2}\right) \eta_{1}^{2 r}\left(\lambda^{2} P^{2}+R^{2}\right)}<0
$$

gives the admissible linear contact coefficient $\lambda>0$, where $Q R-P^{2}>0, Q>0, R>0, P<0$ have been used.

In the permissible range of linear contact coefficient, the relation between the singularity index and the linear contact coefficient is plotted in Fig. 2 for different values of crack velocity. From Fig. 2, one can see the singularity decreases as the linear contact coefficient increases. As a limiting case, when the linear contact coefficient tends to be infinite, the singularity approaches zero.

\section{Finite Contact Case}

In this section, the case of a finite contact zone $l$ on the crack face is investigated. The contact conditions in equations (10) hold within the contact zone, while the traction-free condition should be used for the crack face outside the contact zone, i.e.,

$$
\begin{array}{lrl}
\sigma_{12}=\lambda \sigma_{22} & u_{2}=0 \quad \sigma_{22} \leq 0 & \text { at } \quad-l<\eta_{1}<0, \quad \eta_{2}=0 \\
\sigma_{12}=0 & \sigma_{22}=0 & \text { at } \quad \eta_{1}<-l, \quad \eta_{2}=0
\end{array}
$$

The contact conditions in equation (22) become identical to those in equations (12) in terms of function $\mathbf{h}(z)$, except that they hold only in the region of $-l<\eta_{1}<0$ for this finite contact problem. 


$$
\mathbf{W} \boldsymbol{\theta}^{\prime+}+\mathbf{K} \boldsymbol{\theta}^{\prime-}=0 \quad \mathbf{W}=\left[\begin{array}{cc}
R & P i \\
-P i & Q
\end{array}\right] \quad \mathbf{K}=\left[\begin{array}{cc}
R & -P i \\
P i & Q
\end{array}\right] \quad \eta_{1}<-l
$$

Combining equation (12) and (24) leads to a set of Riemann-Hilbert equations for $\eta_{1}<0$

$$
\left\{\begin{array}{lc}
\mathbf{U} \boldsymbol{\theta}^{\prime+}+\mathbf{V} \boldsymbol{\theta}^{--}=0 & -l<\eta_{1}<0 \\
\mathbf{W} \boldsymbol{\theta}^{\prime+}+\mathbf{K} \boldsymbol{\theta}^{\prime-}=0 & \eta_{1}<-l
\end{array}\right.
$$

However, the discontinuity conditions on the crack face are given separately inside the contact zone and outside the zone as in equations (25). To solve the Riemann-Hilbert equations, we first assume equations (12) hold in the whole region $\eta_{1}<0$, then we obtain the solution as equations (14).

Substituting equations (14) into the second equation in (25) and regarding the functions $A(z)$ and $E(z)$ no longer entire functions now, we can obtain Hilbert equations about $A(z)$ and $E(z)$. Solving the corresponding Hilbert equations and noting that $A(z)$ and $E(z)$ need to be continuous in the region of $-l<\eta_{1}<0$, we obtain the expressions of $A(z)$ and $E(z)$ after tedious derivations,

$$
\left\{\begin{array}{l}
A(z) \\
E(z)
\end{array}\right\}=\left\{\begin{array}{l}
\frac{e(z)}{(z+l)^{1}}+\frac{d(z)}{(z+l)^{\prime 2}} \\
\frac{c_{1}|z|^{r} e(z)}{c_{2}(z+l)^{1}}+\frac{d_{1}|z|^{r} d(z)}{d_{2}(z+l)^{\prime 2}}
\end{array}\right\}
$$

where

$$
\begin{array}{lc}
r_{1}=\frac{1}{2 \pi i} \ln \frac{\frac{\lambda i P Q}{R}-Q+\frac{i P c_{1} e^{-\pi i r}}{c_{2}}}{\frac{\lambda i P Q}{R}+Q+\frac{i P c_{1} e^{\pi i r}}{c_{2}}}, & r_{2}=\frac{1}{2 \pi i} \ln \frac{\lambda Q+i P+\frac{R d_{1} e^{-\pi i r}}{d_{2}}}{-\lambda Q+i P-\frac{R d_{1} e^{\pi i r}}{d_{2}}} \\
c_{1}=(\lambda+R) Q\left(1-a_{1}\right)+i P\left(1+a_{1}\right)(1-\lambda Q), & c_{2}=R(i P-1) e^{-\pi i r}+(i P+1) R a_{1} e^{\pi i r} \\
d_{1}=(\lambda+R) Q\left(1-a_{2}\right)+i P\left(1+a_{2}\right)(1-\lambda Q), & d_{2}=R(i P-1) e^{-\pi i r}+(i P+1) R a_{2} e^{\pi i r} \\
a_{j}=\frac{-b \pm \sqrt{b^{2}-4\left(P^{2}-Q R\right)^{2} e^{2 \pi i r}}}{2\left(P^{2}-Q R\right) e^{2 \pi i r}}, & b=\left(P^{2}+Q R\right)\left(1+e^{2 \pi i r}\right)+2 \lambda i P Q\left(1-e^{2 \pi i r}\right)
\end{array}
$$

It can be proved that ratios of $c_{1} / c_{2}$ and $d_{1} / d_{2}$ are real as well as $r_{1}$ and $r_{2} \cdot e(z)$ and $d(z)$ are entire functions, which are analytical in the whole plane including the crack face.

From the numerical results, we know that $r_{2} \rightarrow 0$ and $r_{2} \ll<r_{1}$. The terms related to $r_{2}$ will be omitted in the following text. For general $e(z)$, the stress field becomes an infinite series. Here, we only give the asymptotic stress components along the crack line

$$
\sigma_{12}=\frac{2}{Q R-P^{2}}\left[\lambda Q+\frac{c_{1} R}{c_{2}}\right] \frac{e_{0}}{\left(\eta_{1}+l\right)^{r_{1}}}, \quad \sigma_{22}=\frac{2}{Q R-P^{2}} \frac{Q e_{0}}{\left(\eta_{1}+l\right)^{r_{1}}}, \quad \text { at } \eta_{1}>0
$$




$$
\begin{aligned}
& \sigma_{12}=\frac{2}{Q R-P^{2}}\left[\lambda Q+\frac{c_{1} R}{c_{2}} \cos (\pi r)\right] \frac{e_{0}}{\left(\eta_{1}+l\right)^{r_{1}}}, \quad \sigma_{22}=\frac{2}{Q R-P^{2}}\left[Q-\frac{c_{1} P}{c_{2}} \sin (\pi r)\right] \frac{e_{0}}{\left(\eta_{1}+l\right)^{r_{1}}}, \\
& \text { at }-l<\eta_{1}<0 \\
& \sigma_{12}=0, \quad \sigma_{22}=0 \quad \text { at } \quad \eta_{1}<-l
\end{aligned}
$$

It can be found that the relation between the shear and normal stress in the contact region is $\sigma_{12}=\lambda \sigma_{22}$ and equation (33) shows that the traction free conditions outside the contact zone are satisfied.

Based on the assumption of steady-state crack growth, the field of particle velocity can be obtained from the displacement field. The particle velocity on the contact crack face is

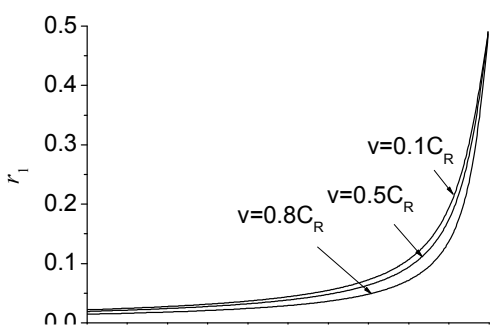

Fig.3 The crack tip singularity via the linear contact coefficient $v_{1}=\frac{2 c_{1} v e_{0} \sin (\pi r)}{c_{2}\left(\left|\eta_{1}\right|+l\right)^{r_{1}}}$

The requirement of the shear traction at the contact crack face resisting crack face sliding yields the range of admissible linear contact coefficient $\lambda$ as $\lambda<0$.

The relation between the linear contact coefficient and the singularity factor is shown in Fig. 3, from where one can see that the singularity decreases sharply from $1 / 2$ when the absolute value of linear contact coefficient increases.

\section{Energy analysis and fracture criterion}

The above analysis shows that the singularity near the crack tip is less than $1 / 2$ which results in a vanishing crack tip energy release rate and reaches $1 / 2$ when the linear contact coefficient tends to be zero. A mechanism of energy absorption [8] to calculate the energy dissipation over the contact zone per unit crack length is adopted in the present paper to analysis the energy dissipation.

The energy dissipation $D$ over the contact zone per unit crack length is given by

$$
D=\int_{-l}^{0}-\sigma_{12} \frac{\partial u_{1}}{\partial \eta_{1}} \mathrm{~d} \eta_{1}=\frac{4 c_{1} e_{0}^{2} \sin (\pi r)\left[\lambda Q+\frac{c_{1} R \cos (\pi r)}{c_{2}}\right]}{\left(Q R-P^{2}\right) c_{2}} \frac{l^{1-2 r_{1}}}{1-2 r_{1}}
$$

The non-negative energy dissipation requires $\lambda<0$, which is equivalent to the condition that the shear tractions resist crack face sliding. Motivated by [8], we postulate that subsonic crack growth will take place with velocity $v$ at a critical sliding displacement evaluated at the end of the contact zone, i.e.,

$$
u_{1}\left(\eta_{1}=-l, \eta_{2}=0\right)=2 \operatorname{Im}\left\{\theta_{1}\left(\eta_{1}=-l, \eta_{2}=0\right)\right\}=\delta_{c}
$$

where $\delta_{c}$ is a constant depending on the materials and bond properties. 


$$
u_{1}\left(\eta_{1}=-l, \eta_{2}=0\right)=\frac{2 c_{1} e_{0} l^{1-r_{i}} \sin (\pi r)}{c_{2}\left(1-r_{1}\right)}
$$

then, substituting the amplitude $e_{0}$ obtained from equations

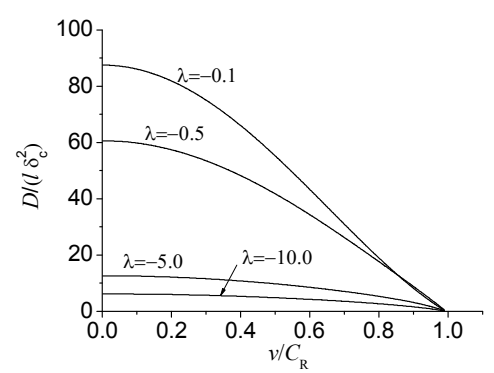

Fig.4 Normalized energy dissipation rate versus the crack velocity
(36) and (37) into equation (35) yields

$$
\frac{D l}{\delta_{c}^{2}}=\frac{\left[c_{2} \lambda Q+c_{1} R \cos (\pi r)\right]\left(1-r_{1}\right)^{2}}{c_{1}\left(Q R-P^{2}\right) \sin (\pi r)\left(1-2 r_{1}\right)}
$$

The dependence of the normalized energy dissipation rate on the crack velocity is plotted in Fig. 4, where the energy dissipation rate $D$ is finite in the range from 0 to near $C_{R}$ and decreases to zero when the velocity approaches $C_{R}$. One should note that the results in the present paper does not adapt to the case that the crack velocity attains

$C_{R}$ exactly.

\section{Acknowledgments}

The work reported here is supported by NSFC (10672165) and KJCX2-YW-M04.

\section{References}

[1] M.L. William: Bull. Seism. Soc. Am. Vol.49 (1959), p. 199.

[2] K.C. Wu: Int. J. Solids Struct. Vol.27 (1991), p.455.

[3] W. Yang, Z. Suo and C.F. Shih: Proc. R. Soc. Lond. A Vol.433 (1991), p.679.

[4] C. Liu, J. Lambros and A.J. Rosakis: J. Mech. Phys. Solids Vol.41 (1993), p.1887.

[5] J. Lambros, and A.J. Rosakis: J. Mech. Phys. Solids Vol.43 (1995), p.169.

[6] X.P. Xu, and A. Needleman: Int. J. Fract Vol.74 (1996), p.289.

[7] X. Deng: J. Mech. Phys. Solids Vol.41 (1993), p.531.

[8] Y. Huang, W. Wang, C. Liu and A.J. Rosakis: J. Mech. Phys. Solids Vol.46 (1998), p.2233.

[9] W. Wang, Y. Huang, A.J. Rosakis and C. Liu: Eng. Fract. Mech. Vol.61 (1998), p.471. 
Advances in Fracture and Materials Behavior

10.4028/www.scientific.net/AMR.33-37

\section{Subsonic Interface Crack with Crack Face Contact}

10.4028/www.scientific.net/AMR.33-37.307

\section{DOI References}

[3] W. Yang, Z. Suo and C.F. Shih: Proc. R. Soc. Lond. A Vol.433 (1991), p.679.

doi:10.1098/rspa.1991.0070

[4] C. Liu, J. Lambros and A.J. Rosakis: J. Mech. Phys. Solids Vol.41 (1993), p.1887.

doi:10.1016/0022-5096(93)90074-P

[5] J. Lambros, and A.J. Rosakis: J. Mech. Phys. Solids Vol.43 (1995), p.169.

doi:10.1016/0022-5096(94)00071-C

[8] Y. Huang, W. Wang, C. Liu and A.J. Rosakis: J. Mech. Phys. Solids Vol.46 (1998), p.2233.

doi:10.1023/A:1007479709559

[9] W. Wang, Y. Huang, A.J. Rosakis and C. Liu: Eng. Fract. Mech. Vol.61 (1998), p.471.

doi:10.1023/A:1007479709559 